\title{
Polarization Transfer in ${ }^{4} \mathrm{He}\left(\vec{e}, e^{\prime} \vec{p}\right)^{3} \mathrm{H}$
}

\author{
Michael Paolone \\ University of South Carolina \\ For the Jefferson Lab Hall-A Collaboration
}

\begin{abstract}
Polarization transfer in quasi-elastic nucleon knockout is sensitive to the properties of the nucleon in the nuclear medium, including possible modification of the nucleon form factor and/or spinor. In our recently completed experiment E03-104 at Jefferson Lab we measured the proton recoil polarization in the ${ }^{4} \mathrm{He}(\overrightarrow{\mathrm{e}}, \mathrm{e} \cdot \mathrm{p})^{3} \mathrm{H}$ reaction at a $\mathrm{Q}^{2}$ of $0.8(\mathrm{GeV} / \mathrm{c})^{2}$ and $1.3(\mathrm{GeV} / \mathrm{c})^{2}$ with unprecedented precision. These data complement earlier data between 0.4 and $2.6(\mathrm{GeV} / \mathrm{c})^{2}$ from both Mainz and Jefferson Lab, in which the measured ratio of polarization-transfer coefficients differs from a fully relativistic DWIA calculation. Preliminary results hint at a possible unexpected $\mathrm{Q}^{2}$ dependence in the polarization transfer coefficient ratio. Final analysis will help constrain FSI models.
\end{abstract}

PACS: $13.40 . \mathrm{Gp} ; 13.88 .+\mathrm{e} ; 24.70 .+\mathrm{s} ; 25.30 . \mathrm{Dh} ; 27.20 .+\mathrm{h}$

The nucleon is a composite object, and while strongly bound inside a dense nucleus one might expect the internal structure to be modified by interaction with external fields. Since the European Muon Collaboration first observed that the ratio of structure functions per nucleon for the deuteron and the dense iron nuclei was not unity $^{1}$, many models of medium modification of the nucleon electromagnetic form factors have been proposed; such as the chiral quark soliton model ${ }^{2}$ (CQSM) or the quark-meson coupling (QMC) model, in which nuclear matter is treated as interacting through self-consistent exchange of scalar and vector mesons ${ }^{3}$. With this model, one can show the predicted ratio of Sachs form factors $\left(\mathrm{G}_{\mathrm{E}} / \mathrm{G}_{\mathrm{M}}\right)$ for a bound nucleon in nuclear medium versus the same form factor ratio for a free unbound nucleon. The QMC model predicts this double-ratio to be dependent on the $Q^{2}$ value of the reaction as well as on the density of the nuclear media. Experimentally, one can extract the ratio of Sachs form factors for a free proton in the polarized scattering reaction ${ }^{1} \mathrm{H}(\overrightarrow{\mathrm{e}}, \mathrm{e} \cdot \overrightarrow{\mathrm{p}})$ by measuring the ratio of transverse and longitudinal polarization-transfer coefficients, $P^{\prime} x / P^{\prime} z$, of the recoiling proton ${ }^{4}$.

The polarization transfer ratio for quasi-elastic scattering in ${ }^{4} \mathrm{He}(\overrightarrow{\mathrm{e}}, \mathbf{e} \cdot \overrightarrow{\mathrm{p}})^{3} \mathrm{H}$ is expected to be sensitive to the ratio of form factors of the bound proton, and is therefore an ideal testing ground for proposed medium modification models. The results for this ratio from previously completed experiments at Mainz ${ }^{5}$ and E93-049 at Jefferson $\mathrm{Lab}^{6}$ hinted at a deviation of form factor ratios for the bound versus the free proton, and indicated the need for higher statistics and additional measurements at intermediate $Q^{2}$ values. Data from these previous experiments differ from a fully relativistic distorted-wave impulse approximation (RDWIA) calculation. This RDWIA calculation including in-medium modifications as predicted by QMC provide a closer

CP947, VII Latin American Symposium on Nuclear Physics and Applications

edited by R. Alarcon, P. L. Cole, C. Djalali, and F. Umeres

(C) 2007 American Institute of Physics 978-0-7354-0461-8/07/\$23.00 
description of the data. In addition, the data are also well described by calculations by Schiavilla ${ }^{7}$, which include a charge exchange in the final state interaction. While measurement of the polarization transfer to the recoil proton is essential in ${ }^{4} \mathrm{He}(\overrightarrow{\mathrm{e}}, \mathrm{e} \cdot \vec{p})^{3} \mathrm{H}$ for studying the relative strength of medium modification; measuring the induced polarization of the recoil proton provides insight into the strength of final state interactions (FSI). E03-104 was recently completed at Jefferson Lab as a follow up experiment to E93-049, providing higher statistics at intermediate $Q^{2}$ points.

Jefferson Lab's experimental Hall-A includes two high-resolution spectrometer (HRS) $\mathrm{arms}^{8}$; for E03-104, a polarized electron beam incident on both ${ }^{4} \mathrm{He}$ and ${ }^{1} \mathrm{H}$ targets produced scattered electrons and protons each then detected per reaction in coincidence. The incident beam energy was $2.0 \mathrm{GeV}$ and $2.6 \mathrm{GeV}$ for $\mathrm{Q}^{2}$ values of $0.8(\mathrm{GeV} / \mathrm{c})^{2}$ and $1.3(\mathrm{GeV})^{2}$ respectively, and the beam was maintained at an average beam polarization of approximately $85 \%$. The proton HRS was also equipped with a focal plane polarimeter (FPP) to measure both the transferred and induced recoil proton polarization'.

Preliminary analysis of the E03-104 ${ }^{1} \mathrm{H}\left(\overrightarrow{\mathrm{e}}, \mathrm{e}^{\prime} \overrightarrow{\mathrm{p}}\right)$ reaction indicates statistical uncertainties at least three times smaller than E93-049, and show good agreement with world data. For ${ }^{4} \mathrm{He}\left(\overrightarrow{\mathrm{e}}, \mathrm{e}^{\prime} \overrightarrow{\mathrm{p}}\right)^{3} \mathrm{H}$, figure 1 shows $\mathrm{R} / \mathrm{R}_{\text {PwIA }}$ versus $\mathrm{Q}^{2}$ where $\mathrm{R}$ is the ratio of polarization transfer coefficients for the bound proton versus free proton. $R_{P W I A}$ is a baseline calculation using the plane wave impulse approximation. Shown are data from MAMI and E93-049, as well as preliminary data from E03-104. For E03-104's data, the systematic error is expected to be reduced in the final analysis by no less than a factor of two. The data are compared to calculations by Udias ${ }^{10}$ with (solid curve) and without (dashed curve) including medium modifications predicted by the QMC model $^{3}$, as well as a calculation by Schiavilla ${ }^{7}$ including, particularly, charge-exchange final state interactions and two-body charge and current operators (MEC). The data is better described by the RDWIA calculations including in-medium modified predictions than without, but are also well described by Schiavilla's calculations including FSI. The relatively low $\mathrm{R}$ value for the $0.8(\mathrm{GeV} / \mathrm{c})^{2}$ suggests a possible $\mathrm{Q}^{2}$ dependence not predicted by our model calculations.

In figure 2 we have plotted the induced proton polarization normal to the scattering plane $\left(\mathrm{P}_{\mathrm{y}}\right)$ versus $\mathrm{Q}^{2}$. Plotted again are Schiavilla's calculation, and the Udias calculations with and without medium modification predictions. Here the data is best described by the Udias' calculations; $\mathrm{P}_{\mathrm{y}}$ is not very sensitive to in-medium form factors but is a measure of FSI effects, and the latter are not well constrained in the energy range of this experiment. The data indicate a possible over-estimation of the charge-exchange FSI in Schiavilla's calculation. Systematic uncertainties shown are larger than those expected for the final analysis due to the presence of instrumental asymmetries in the FPP.

In conclusion, the polarization transfer technique is a powerful tool to probe the strength of medium modification. Prior data taken at MAMI and JLab have shown a need for higher statistics and data at intermediate $Q^{2}$ values. The recently completed experiment E03-104 at JLab is currently being analyzed; preliminary results hint at a possible $Q^{2}$ dependence in the ratio of polarization transfer coefficients for the free versus the bound proton, and final analysis will help constrain the description of FSI in models. 


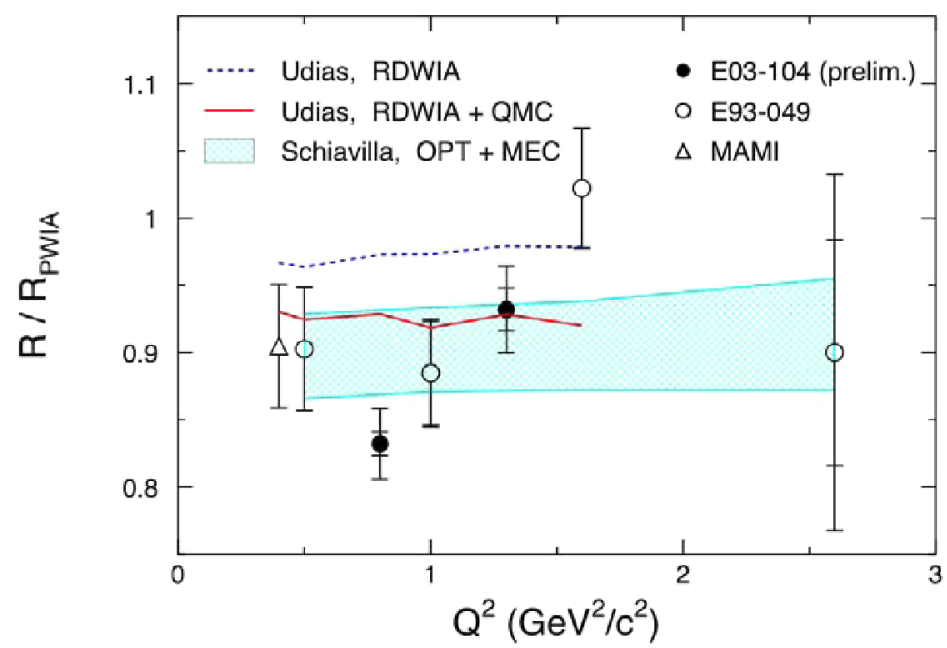

FIGURE 1. Polarization-transfer data from Mainz $z^{5}$ and $\mathrm{JLab}^{6}$ along with preliminary data from experiment E03-104. $\mathrm{R}$ is the ratio of transverse to longitudinal polarization of the recoiling protons in ${ }^{4} \mathrm{He}\left(\overrightarrow{\mathrm{e}}, \mathrm{e}^{\prime} \overrightarrow{\mathrm{p}}\right)^{3} \mathrm{H}$ compared to the same ratio for ${ }^{1} \mathrm{H}(\overrightarrow{\mathrm{e}}, \mathrm{e} \cdot \overrightarrow{\mathrm{p}})$. Not shown is the Ghent RDWIA calculation ${ }^{11}$. Please refer to text for discussion of models.

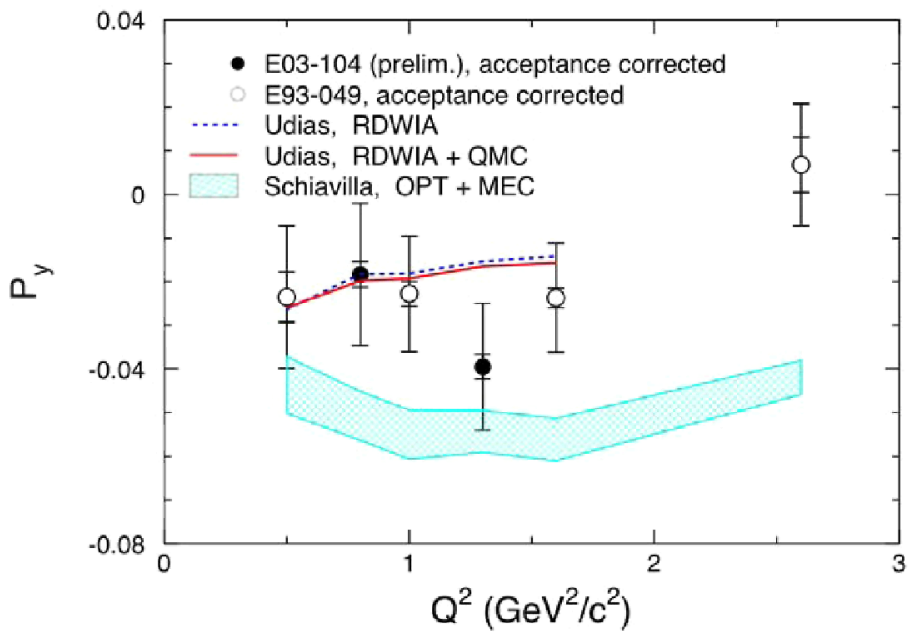

FIGURE 2. Induced polarization in the $y$-direction normal to the scattering plane as a function of $Q^{2}$. Data from E93-049 and preliminary data from E03-104 are shown; both are acceptance corrected. Refer to text for discussion of models. 


\section{ACKNOWLEDGMENTS}

This work was supported in parts by the U.S. National Science Foundation: NSF PHY-0555604.

Jefferson Science Associates operates the Thomas Jefferson National Accelerator Facility under DOE contract DE-AC05-06OR23177.

\section{REFERENCES}

1. J.Aubert et al., Phys. Lett. B 123, 275 (1983).

2. J.R. Smith and G.A. Miller, Phys. Rev. Lett. 91, 212301 (2003)

3. D. H. Lu et al., Phys. Lett. B 417, 217 (1998): Phys. Rev. C 60, 068201 (1999).

4. A.I. Akhiezer and M.P. Rekalo, Sov. J. Part. Nucl 3, 277 (1974);

R. Arnold, C. Carleson and F. Gross, Phys. Rev. C 23, 363 (1981).

5. S. Dieterich et al., Phys. Lett. B 500, 47 (2001).

6. S. Strauch et al., Phys. Rev. Lett. 91, 052301 (2003).

7. R. Schiavilla et al., Phys. Rev. Lett. 94, 072303 (2005).

8. J. Alcorn et al., Nuc. Instrum. and Methods A522, 294 (2004).

9. V. Punjabi, Phys. Rev. C 71, 055202 (2005).

10. J.M.Udias, J.R. Vignote, Phys. Rev. C 62 034302(2000).

11. P. Lava et al., Phys. Rev. C 71, 014605 (2005). 